\title{
A Community-Based Approach to Enhancing Anal Cancer Screening in Hawaii's HIV-Infected Ethnic Minorities
}

Bruce Shiramizu $^{1 *}$, Cris Milne', Kevin Terada ${ }^{1}$, Kevin Cassel ${ }^{2}$, Rayna K. Matsuno ${ }^{2}$, Jeffery Killeen ${ }^{3}$, Chin-Yuan Liang ${ }^{1}$, Faye Tachibana ${ }^{1}$, Tom Sheeran ${ }^{4}$, James Weihe ${ }^{4}$ and Marc T. Goodman ${ }^{2}$

${ }^{1}$ John A. Burns School of Medicine, University of Hawaii, Honolulu, Hawaii, USA

${ }^{2}$ Cancer Center, University of Hawaii, Honolulu, Hawaii, USA

${ }^{3}$ John A. Burns School of Medicine, Department of Pathology, University of Hawaii, Honolulu, Hawaii, USA

${ }^{4}$ Hawaii Center for AIDS Community Advisory Board, Honolulu, Hawaii, USA

\begin{abstract}
Objective: Disparities in anal cancer incidence among Hawaii's HIV-infected minority population is an emerging health concern. Although anal cytology/anoscopy are effective anal cancer screening tools, social barriers exist that prevent individuals from seeking appropriate care.

Design: Community based participatory research (CBPR) principles were applied to develop resources, including testing a self-obtained anal specimen procedure, to increase anal cancer screening among Hawaii's underserved/ minority populations.

Methods: A team of community members, academic researchers, and health care providers developed culturally-sensitive educational/recruitment materials regarding anal cancer risk targeting underserved/minority HIVinfected individuals. Self- and health care provider (HCP)-obtained anal cancer screening specimens were reviewed for cytology and tested for human papillomavirus DNA. A follow-up evaluation elicited feedback on attitudes and experiences.
\end{abstract}

Results: Community discussion sessions identified key messages about anal cancer, anal cancer screening, and HPV infection for materials and were used, that successfully recruited 46 individuals ( 38 males/8 females; 9 Native Hawaiians/Pacific Islanders/Asians, 2 Blacks, 6 Hispanics, 6 American Indian/Alaskan Natives, 23 Whites). Concordance in cytology results between self- and HCP-obtained specimens was moderated (kappa=0.37) with the perception that the self-obtained specimen procedure was private $(93 \%)$, safe $(100 \%)$, and easy to manage $(100 \%)$; and a majority $(92 \%)$ willing to use the self-obtained method again.

Conclusions: CBPR was a practical approach in engaging Hawaii's HIV-infected minority participation in anal cancer screening research. Community outreach and recruitment efforts suggested that self-obtained screening specimens could be an acceptable and effective means to reach Hawaii's HIV-infected ethnic minorities.

Keywords: Human papillomavirus; Anal neoplasia; Anal dysplasia; Human immunodeficiency virus

\section{Introduction}

Anal cancer rates have risen in the past three decades for both men and women $[1,2]$ with a five-fold increased risk among HIVseropositive men and women [3,4]. Like cervical cancer, the majority of anal cancers are associated with human papillomavirus (HPV) infection [5-10]. The incidence of anal cancer in men who have sex with men (MSM) before the onset of the HIV epidemic was approximately 35 per 100,000 , comparable to cervical cancer incidence in women before the introduction of cervical cytology screening [3,4,10-12]. It is therefore likely that the early detection of anal cancer or anal intraepithelial lesions (AIN) in high-risk, HIV-infected individuals would have tangible public health benefits.

There continue to remain barriers to anal cancer screening within high-risk populations including the need for education about the importance of anal cytology screening among health care providers and their patients, particularly in Hawaii where the HIV/AIDS epidemic disproportionately affects ethnic minorities [13,14]. In Hawaii, the stigma of HIV/AIDS in the Native Hawaiian-Pacific Islander and Asian population remains a major obstacle to seeking appropriate healthcare which is compounded by the segmented Hawaiian Islands' geography, general physician shortages, and rural isolated communities [15].

The Hawaii Center for AIDS with its Community and Scientific
Advisory Boards, representing patients, medical providers, patient advocates and AIDS service organizations and patient navigator determined that information on the importance and availability of anal cancer screening was not being effectively disseminated to Hawaii's underserved Native Hawaiian-Pacific Islanders and Asians at risk for anal cancer. Given this need for education and access to preventive care for anal cancer, the current study was designed to support collaborations between clinical researchers, medical providers, and members of Hawaii's HIV infected community to meet the following three primary research objectives: 1) To enhance community awareness on the importance of anal cancer screening among HIV-infected Native Hawaiian-Pacific Islanders and Asians; 2) To evaluate HPV DNA and anal cytologies from self-obtained and healthcare provider (HCP)-

*Corresponding author: Bruce Shiramizu, MD, Departments of Pediatrics, Medicine and Tropical Medicine, John A. Burns School of Medicine, 651 llalo St. BSB 325, Honolulu, Hawaii, USA, Tel: (808) 692-1677; Fax: (808) 692-1984; E-mail: bshirami@hawaii.edu

Received June 15, 2012; Accepted July 10, 2012; Published July 13, 2012

Citation: Shiramizu B, Milne C, Terada K, Cassel K, Matsuno RK, et al. (2012) A Community-Based Approach to Enhancing Anal Cancer Screening in Hawaii's HIVInfected Ethnic Minorities. J AIDS Clinic Res 3:162. doi:10.4172/2155-6113.1000162

Copyright: (c) 2012 Shiramizu B, et al. This is an open-access article distributed under the terms of the Creative Commons Attribution License, which permits unrestricted use, distribution, and reproduction in any medium, provided the original author and source are credited. 
obtained specimens to compare the two specimen collection methods; and 3) To determine whether a self-obtained anal specimen procedure might reduce barriers to anal cancer screening within Hawaii's multiethnic and multicultural population. The diverse ethnicities found in Hawaii and the cultural sensitivities of these populations regarding HIV infection necessitated the identification of specific, sensitive, and appropriate communication strategies to build broad awareness about anal cancer risks and screening. We hypothesized that self-obtained cytology specimens would minimize a patient's self-consciousness and fear of discomfort associated with anal specimen collection. While previous studies demonstrated that self-obtained cytology samples from MSM were adequate for cytological examination and comparable in quality to those obtained from clinicians $[16,17]$, the demographic profile of the 'at-risk' population in Hawaii diverged substantially from participants in previous studies who were already accustomed to routine cytological testing or were mainly Caucasians $[16,17]$. This study sought to understand patients' perceptions and attitudes concerning self-obtained cytology samples as compared with those collected by a health care provider.

\section{Methods}

\section{Community-based participatory research (CBPR)}

CBPR methods were used to promote mobilization and education within Hawaii's HIV- infected community about anal cancer risks and the adoption of anal cancer screening [18]. The research team was comprised of representatives from the University of Hawaii Cancer Center and the Hawaii Center for AIDS and the patient navigator as part of the NCI-funded AIDS Malignancy Consortium. This group convened the Hawaii Center for AIDS Community and Scientific Advisory Boards as a first step towards engaging Hawaii's HIV-infected community with the study [18]. The Community and Scientific Advisory Board study team consisted of twelve members that included HIV-infected patients, community physicians, public policy personnel, and health care professionals caring for HIV-infected clients in Hawaii. The Community and Scientific Advisory Boards were previously established to provide advice and input for other clinical translational research involving HIV-infected patients. These combined groups of researchers, clinical providers, patient navigator, and members of Hawaii's HIV-infected community served as the research study team [18].

The community-academic research team met bi-annually throughout the course of the study. During the first meetings, the community-academic team served as key-informants to identify salient messages that could be used to inform the community about anal cancer screening and which could be used to recruit participants to the study [19]. A list of potential anal cancer educational messages was developed from a review of the scientific literature and from current patient education resources used at clinics elsewhere and were reviewed by the research team selecting a set using nominal group process [20]. A survey was developed and administered to the research study team members to evaluate the selected messages with HIV-infected individuals in the community [21].

A guided discussion session was subsequently held with HIVinfected individuals at the Life Foundation, a non-profit AIDS-service organization assisting people who are living with HIV and AIDS in Hawaii [19]. These sessions were designed to evaluate information forwarded by the community-academic research team with HIVinfected individuals in Hawaii about: 1) the importance of anal cancer screening; 2) the use of anal cytology for screening, and; 3) the key educational messages selected to promote anal cancer screening and recruitment. Analysis of survey data was conducted using SPSS Version 19 (SPSS, Inc., 2010, Chicago, IL). Discussion group data was coded and analyzed using Grounded Theory [22]. The study was reviewed and approved by the University of Hawaii Institutional Review Board and patients underwent an informed consent process prior to participation.

\section{Participant recruitment and specimen collection for anal cytology}

Recruitment of participants was accomplished by organizing information sessions at community forums, through recruitment materials at public events and clinic offices, through information dissemination by the patient navigator at neighbor island AIDS service organizations, and referrals by community physicians. Participants were recruited to compare self-obtained- versus HCP-collected anal specimens for cytology during the same research clinic visit using a modified protocol [23]. Consented participants who met the inclusion criteria (HIV-seropositive, male or non-pregnant female $\geq 18$ years old, ability to perform daily activities) collected an anal cytology specimen himself or herself following instructions provided by the HCP who was not present during the self-obtained procedure. The HCP subsequently collected a second anal cytology specimen, as well as additional research specimens. The self-collection process included a kit consisting of a tube containing CytoThinPrep sample collection medium (Cytyc Corp., Boxborough, MA), a Dacron swab that was prepared with a marking tape on the swab handle 3 inches from the tip, and latex gloves. After applying gloves, the subject moistened the Dacron swab with tap water in a cup before inserting it 2-3 inches into the anal canal. A taped marking on the handle was used to indicate the three inch limit. With gentle pressure to the walls of the anal canal, the patient withdrew the swab from the anal canal with a spiral motion over a 10-second period and placed the swab into the CytyoThinPrep medium. The swab was agitated vigorously in the medium for 10 seconds and discarded in an appropriate waste container and the collection tube covered. The HCP repeated the same procedure with the second swab placed in a separate medium container. Both containers were sent to a CLIA-certified laboratory, processed, and the cytology reviewed by the same pathologist (JK) according to the Bethesda system and anal intraepithelial neoplasia (AIN) system [24,25]; and categorized as: 1) Negative if no cellular changes could be detected or if cellular changes were caused by inflammation or reparative process; or 2) Low- or high grade squamous intraepithelial lesions (LGSIL/HGSIL) for simple HPV changes and AIN I or AIN II and III, respectively. Concordance was defined if the HCP- and subject-obtained specimens which had similar AIN I/II/III or negative cytologies.

\section{HPV genotyping}

DNA from a separate anal specimen was extracted using commercial reagents (Qiagen Inc, Valencia CA) and analyzed for the presence or absence of HPV DNA by PCR using a modified version of the PGMY09/PGMY11 primer system [26]. HPV DNA-positive specimens were then genotyped using a reverse line blot detection method for 37 different HPV types including high-risk (HR) types 16, 18, 31, 33, 35, $39,45,51,52,53,56,58,59,66,68,70,73$, and 82; low-risk (LR) types 6 , $11,42,54,61,72,84$, and CP6108; and undetermined risk types 55, 62, $67,71,83$, and 84 (Roche Molecular Systems, Pleasanton, CA) [26-28]. $\mathrm{HPV}$-positive specimens that were subsequently found to be negative in the genotyping assay were considered to be unclassified HPV-positive 


\begin{tabular}{|l|c|c|c|c|c|c|}
\hline & \multicolumn{2}{|c|}{ All } & \multicolumn{2}{c|}{ Males } & \multicolumn{2}{c|}{ Females } \\
\hline & N & $\%$ & $\mathbf{n}$ & $\%$ & $\mathbf{n}$ & $\%$ \\
\hline Number of subjects & 46 & 100 & 38 & 83 & 8 & 17 \\
\hline Mean age (SE) & \multicolumn{2}{|c|}{$49(1.2)$} & \multicolumn{2}{|c|}{49} & $1.4)$ & $50(1.7)$ \\
\hline Race/ethnicity & & & & & & \\
\hline Non-Hispanic White & 23 & 50 & 18 & 47 & 5 & 62 \\
\hline Hispanic/Latino & 6 & 13 & 5 & 13 & 1 & 12 \\
\hline African American & 2 & 4 & 1 & 3 & 1 & 12 \\
\hline Asian & 4 & 9 & 3 & 8 & 1 & 12 \\
\hline Native Hawaiian/Pacific Islander & 5 & 11 & 5 & 13 & 0 & 0 \\
\hline American Indian/Alaska Native & 6 & 13 & 6 & 16 & 0 & 0 \\
\hline HPV test result* & & & & & & \\
\hline Negative & 17 & 37 & 12 & 32 & 5 & 62 \\
\hline Positive & 29 & 63 & 26 & 68 & 3 & 38 \\
\hline Oncogenic HPV genotype & & & & & & \\
\hline Negative & 24 & 52 & 18 & 47 & 6 & 75 \\
\hline Positive & 22 & 48 & 20 & 53 & 2 & 25 \\
\hline HCP-specimen cytology & & & & & & \\
\hline Negative & 28 & 61 & 22 & 58 & 6 & 75 \\
\hline AIN I/I/IIII* & 18 & 39 & 16 & 42 & 2 & 25 \\
\hline Subject-specimen cytology & & & & & & \\
\hline Negative & 26 & 56 & 20 & 53 & 6 & 75 \\
\hline AIN I/I/IIII* & 20 & 44 & 18 & 47 & 2 & 25 \\
\hline
\end{tabular}

Key: n, number; SE, standard error; HPV, Human Papillomavirus; HCP, health care provider

*Positive for any HPV type

tPositive for oncogenic HPV type(s) only

**AIN I/II/III: Anal intraepithelial neoplasia Grade I (mild changes in abnormal cells); Grade II (moderate changes in abnormal cells); Grade III (severe changes in abnormal cells)

Table 1: Participant Demographics.

\begin{tabular}{|c|c|c|c|c|c|c|c|c|}
\hline \multicolumn{2}{|c|}{ A. All subjects } & & \multirow[t]{2}{*}{ B. Males } & & & \multicolumn{3}{|c|}{ C. Females } \\
\hline & HCP C & ytology & & \multicolumn{2}{|c|}{ HCP Cytology } & & \multicolumn{2}{|c|}{ HCP Cytology } \\
\hline $\begin{array}{l}\text { Subject } \\
\text { Cytology }\end{array}$ & Negative & Atypical & $\begin{array}{l}\text { Subject } \\
\text { Cytology }\end{array}$ & Negative & Atypical & $\begin{array}{l}\text { Subject } \\
\text { Cytology }\end{array}$ & Negative & Atypical \\
\hline Negative & 20 & 6 & Negative & 15 & 5 & Negative & 5 & 1 \\
\hline Atypical & 8 & 12 & Atypical & 7 & 11 & Atypical & 1 & 1 \\
\hline \multicolumn{3}{|c|}{$\begin{array}{l}\text { к: } 0.37 \text { (95\% Cl: } 0.10 \text {, } \\
0.64)\end{array}$} & \multicolumn{3}{|c|}{$\begin{array}{l}\text { к: } 0.36 \text { (95\% Cl: } 0.07 \text {, } \\
0.66)\end{array}$} & \multicolumn{3}{|c|}{$\begin{array}{l}\text { к: } 0.33 \text { (95\% Cl: -0.41, } \\
1.00)\end{array}$} \\
\hline
\end{tabular}

Table 2A: Concordance of Anal Cytology Results: Subject versus Health Care Provider (HCP).

specimens. All specimens were tested for the human $\beta$-globin gene as an internal control for sample sufficiency. Specimens testing negative for $\beta$-globin were considered insufficient and excluded from analyses.

\section{Self-obtained anal cytology specimen evaluation}

A questionnaire was administered to assess perceptions about selfobtained anal cytology specimens. The questionnaire was adapted by the community-academic research team from an instrument originally created to assess self-obtained specimens for Chlamydia [29]. Study participants were contacted and those who agreed to participate in the survey were given the questionnaire with arrangements to have the completed survey returned to study personnel.

\section{Statistical analysis}

Concordance between subject- and HCP-collected specimens was evaluated using Cohen's Kappa statistic ( $\kappa$ ) [30]. Logistic regression was used to assess the association between oncogenic HPV genotype and a positive cytology result [31]. Chi-square test was used to analyze by collection methods.

\section{Results}

\section{Community-based participatory research}

Fourteen of the 32 (43\%) discussion session attendees completed the survey that sought to identify key messages about anal cancer, anal cancer screening, and HPV transmission and infection. The themes that were identified confirmed the primary importance of providing information about anal cancer risks and screening to HIV-infected individuals in Hawaii. Key messages were used to create recruitment materials including posters, flyers, and brochures to educate Hawaii's HIV-infected community about the importance of anal cancer screening and to encourage participation in the study. The patient navigator led discussion sessions and assisted participants through the study. These sessions enhanced study recruitment, particularly in Hawaii's rural communities including the neighbor islands.

\section{Anal cancer screening participants}

Forty-six participants were recruited including 38 men and 8 women (Table 1). The patient navigator and community-vetted promotional materials recruited HIV-positive individuals from rural areas and diverse ethnic groups throughout the Hawaii. Six of seven study participants recruited from rural areas in Hawaii joined as a result of outreach efforts by the patient navigator. Participant demographics included Asians (9\%), Native Hawaiians and Pacific Islanders (11\%), Hispanics (13\%), African-Americans (4\%), and American Indians and Alaskan Natives (13\%). Seven participants (15\%) resided in neighboring (non-Oahu) Hawaiian Islands (Table 1). All of the subjects participated in the self- and HCP-obtained anal collection process.

\section{Anal cytology and HPV genotyping}

The anal cytology results from HCP- versus self-obtained specimens showed moderate concordance, $\kappa=0.37$ with no difference in concordance between genders (Table $2 \mathrm{~A}$ ). Concordance was defined if both HCP- and subject-obtained specimens had similar AIN I/II/ III or negative cytology results. For HPV genotyping, all specimens initially screened for good quality DNA using $\beta$-globin primers. HCP- and subject-collected anal cytologies with AIN I/II/III showed moderate concordance for the presence of oncogenic HPV in the same specimens, $\kappa=0.31$, with HCP-obtained AIN I/II/III specimens being 3.6-fold more likely to be associated with an oncogenic HPV type than specimens with a normal cytology $(\mathrm{p}=0.04)$ (Table $2 \mathrm{~B})$. The $\mathrm{p}$-value tests whether the odds ratio (OR) is statistically significantly different from 1.0 (null). A p-value of less than 0.05 indicates that there is a statistically significant association for the different oncogenic types versus the reference group. In contrast, among subject-obtained specimens, no significant association of AIN I/II/III with oncogenic

\begin{tabular}{|c|c|c|c|c|}
\hline A. HCP-Obtained Specimens & $\mathbf{n}$ & OR & $95 \% \mathrm{Cl}$ & p-value ${ }^{* * *}$ \\
\hline Non-oncogenic HPV only / negative & 24 & 1 & $\begin{array}{l}\text { Reference } \\
\text { group }\end{array}$ & \\
\hline Any oncogenic HPV type(s) & 22 & 3.6 & $1.0,12.5$ & 0.04 \\
\hline B. Subject-Obtained Specimens & $\mathbf{n}$ & OR & $95 \% \mathrm{Cl}$ & p-value $e^{* * \star}$ \\
\hline Non-oncogenic HPV only / negative & 24 & 1 & $\begin{array}{l}\text { Reference } \\
\text { group }\end{array}$ & \\
\hline Any oncogenic HPV type(s) & 22 & 2.4 & $0.7,7.9$ & 0.15 \\
\hline C. HCP or Subject-Obtained Specimens & $\mathbf{n}$ & OR & $95 \% \mathrm{Cl}$ & p-value $e^{* * *}$ \\
\hline Non-oncogenic HPV only/negative & 24 & 1 & $\begin{array}{l}\text { Reference } \\
\text { group }\end{array}$ & \\
\hline Any oncogenic HPV type(s) & 22 & 3.7 & $1.1,12.9$ & 0.04 \\
\hline
\end{tabular}

Table 2B: Association of AIN I/II/III* with the Risk of Oncogenic HPV Genotype by $\mathrm{HCP}^{* *}$ - or Subject-Obtained Specimen. 


\begin{tabular}{|l|c|c|c|c|}
\hline A. HCP-Obtained Specimens & $\mathbf{n}$ & OR & $\mathbf{9 5 \%} \mathbf{C l}$ & p-value $^{* * *}$ \\
\hline Non-HPV-16/18 or negative & 33 & 1 & Reference group & \\
\hline HPV-16 and/or -18 & 13 & 3.7 & $1.0,14.1$ & 0.06 \\
\hline B. Subject-Obtained Specimens & $\mathbf{n}$ & OR & $\mathbf{9 5 \%} \mathbf{C l}$ & p-value $^{* * *}$ \\
\hline Non-HPV-16/18 or negative & 33 & 1 & Reference group & \\
\hline HPV-16 and/or -18 & 13 & 2.8 & $0.7,10.5$ & 0.13 \\
\hline C. HCP- or Subject-Obtained Specimens & $\mathbf{n}$ & OR & $\mathbf{9 5 \%}$ Cl & p-value $^{* * *}$ \\
\hline Non-HPV-16/18 or negative & 33 & 1 & Reference group & \\
\hline HPV-16 and/or -18 & 13 & 6.6 & $1.3,34.5$ & 0.02 \\
\hline
\end{tabular}

*AIN I/II/III: Anal intraepithelial neoplasia Grade I (mild changes in abnormal cells); Grade II (moderate changes in abnormal cells); Grade III (severe changes in abnormal cells)

${ }^{* *} \mathrm{HCP}$ : healthcare provider

*** $\mathrm{p}$-value tests whether the odds ratio $(O R)$ is statistically significantly different from 1.0 (null); a p-value of $<0.05$ suggests that the association for the different oncogenic types versus the reference group is statistically significant

Table 2C: Association of AIN I/II/III with Risk of HPV-16 and/or -18.

HPV was found; although for any AIN I/II/III specimen regardless of who collected the specimen there was a 3.7-fold increased likelihood of association with an oncogenic HPV type $(\mathrm{p}=0.04)$ (Table 2B). Similar findings were noted when the analyses were focused on AIN I/II/III and only oncogenic HPV genotypes 16 and 18 (Table 2C).

\section{Self-obtained anal cytology specimen evaluation}

Among the 46 participants, 31 (67\%) consented to complete the follow-up questionnaire. Participants preferred to be evaluated in the Hawaii Center for AIDS clinic (42\%) or given the option of having a self-obtained (45\%) anal cancer screening procedure rather than to have an anal screening test performed by their private physician (13\%). This preference was not influenced by concordance with the HCP-obtained sample (overall, $\mathrm{p}=0.80$ ) or with the presence of oncogenic HPV (overall, $\mathrm{p}=0.39$ ). There was also no difference in preference between the patient preferring to obtain his/her own specimen versus someone else (either the HCP or private physician), $\mathrm{p}=0.40$. However, given the three options of collecting the anal specimen (self, HCP, private physician), self-obtain collection was preferred, $\mathrm{p}=0.01$, but the small sample size limits the interpretation of the analysis. No differences were observed in preference by who obtained the anal specimen based on gender $(\mathrm{p}=0.81)$ or by ethnic minority background $(\mathrm{p}=0.86)$. There was a trend towards non-Caucasians finding the self-obtained collection more comfortable compared to Caucasians; and overall, the age group 40-60 years described the self-obtained procedure to be uncomfortable.

The self-obtained anal cytology specimen procedure was perceived by participants as being private (93\%), safe (100\%), and easy to manage (100\%), regardless of concordance with the HCP-obtained sample or the HPV result. The majority of participants (92\%) stated that they would use the self-obtained anal specimen collection method again and would prefer to collect their own specimen even if the results were inconsistent with those obtained by the HCP or were found to be HPV positive. To determine if any of the preferences were different based on ethnicity was not possible because the post-study survey was administered without any asking for any personal identifying information.

\section{Discussion}

Community engagement and input provided an opportunity to disseminate culturally-appropriate and culturally-sensitive information to complement recruitment efforts by the patient navigator in the anal cancer screening study among HIV-infected Native Hawaiians, Pacific Islanders, Asians, disadvantaged, and rural populations in Hawaii including populations on neighboring Hawaiian Islands. The rich ethnic and racial diversity in Hawaii set the foundation to implement CBPR principles for enhancing anal cancer screening among HIV-infected minority populations. To achieve our objectives community members, AIDS service organizations, health care practitioners, the Hawaii State Department of Health and other community stakeholders assisted with the study's implementation and promotion. Engagement of community advisory groups and Hawaii's AIDS service organizations allowed for interactive feedback which fostered a sense shared responsibilities that served to support and refine the study design, methods and resources, and facilitated the dissemination of research findings. The action orientation of the study enhanced anal cancer screening among HIVinfected men and women while building the knowledge and skills of community members and researchers.

Our results demonstrated substantial ethnic minority participation in anal cancer screening, including active involvement from rural Hawaiian island communities that have been traditionally underserved. Community outreach with the patient navigator visiting the neighbor island AIDS service organization disseminated information about the importance of anal cancer screening. Among the seven study participants from Oahu's neighbor islands, six had their first anal cancer screening through enrollment in the study.

A long-term research objective was to design an anal cytology self-collection home kit for HIV-infected populations in Hawaii who continue to experience barriers to anal cancer screening in a healthcare setting. The results of the current study demonstrated reasonable concordance between subject- and HCP-obtained anal cytology specimens, although the agreement in our study was lower than anticipated from published research [16,23]. One possible explanation for the less than expected concordance between cytology results from subject- versus HCP-obtained anal specimens is that the anal cancer screening was the first experience for $72 \%$ of the participants and the limited exposure and experience on the methodology and process. Laboratory differences in interpreting cytology results are less likely sources of variation because the same pathologist (JK) reviewed all of the cases.

The follow-up survey of the study participants demonstrated overwhelming satisfaction with the ease of the self-obtained anal cytology specimen collection method. There was also no significant difference in whether patients preferred to obtain their own specimen versus having a specimen obtained by either the HCP in the research clinic or their private physician. These data served to design a follow up study of a self-obtained home sampling kit that would be an option for anal cancer screening that could minimize an individual's consciousness and fear of discomfort associated with anal cytology collection in the clinical setting. The small sample size is a limitation of the study and validation of the results is needed in a larger study. The study was also not designed to evaluate the cost-effectiveness of implementing selfobtained anal cytology collection. This would be important to assess in future studies.

Native Hawaiians account for an increasing proportion of AIDS cases in Hawaii, thus education and information dissemination regarding HIV-associated anal cancer and its relation to HPV infection is an emerging public health priority [32,33]. Our results underscore the potential of the CBPR approach to increasing anal cancer screening among Hawaii's underserved populations. The study results demonstrated that building alliances with key community partners to engage populations at high-risk for anal malignancy was effective in disseminating information and participating in anal cancer screening of Hawaii's HIV-infected community. 
Citation: Shiramizu B, Milne C, Terada K, Cassel K, Matsuno RK, et al. (2012) A Community-Based Approach to Enhancing Anal Cancer Screening in Hawaii's HIV-Infected Ethnic Minorities. J AIDS Clinic Res 3:162. doi:10.4172/2155-6113.1000162

\section{Acknowledgements}

Special thanks are extended to the participants of the study, the Life Foundation, the Maui AIDS Foundation, and other community partners.

\section{Grant Support}

The study was supported by grants CA096254, CA143727, CA121947, CA077318, RR026136, and MD007584.

\section{References}

1. Clark MA, Hartley A, Geh JI (2004) Cancer of the anal canal. Lancet Oncol 5: 149-157.

2. Johnson LG, Madeleine MM, Newcomer LM, Schwartz SM, Daling JR (2004) Anal cancer incidence and survival: the surveillance, epidemiology, and end results experience, 1973-2000. Cancer 101: 281-288.

3. Crum-Cianflone NF, Hullsiek KH, Marconi VC, Ganesan A, Weintrob A, et al (2010) Anal cancers among HIV-infected persons: HAART is not slowing rising incidence. AIDS 24: 535-543.

4. Patel P, Hanson DL, Sullivan PS, Novak RM, Moorman AC, et al. (2008) Incidence of types of cancer among HIV-infected persons compared with the general population in the United States, 1992-2003. Ann Intern Med 148: 728736

5. Franceschi S, De Vuyst $H$ (2009) Human papillomavirus vaccines and anal carcinoma. Curr Opin HIV AIDS 4: 57-63.

6. Giuliano AR, Tortolero-Luna G, Ferrer E, Burchell AN, de Sanjose S, et al. (2008) Epidemiology of human papillomavirus infection in men, cancers other than cervical and benign conditions. Vaccine 26: K17-28.

7. Kreuter A, Brockmeyer NH, Altmeyer P, Wieland U; German Competence Network HIVIAIDS (2008) Anal intraepithelial neoplasia in HIV infection. J Dtsch Dermatol Ges 6: 925-934.

8. Galani E, Christodoulou C (2009) Human papilloma viruses and cancer in the post-vaccine era. Clin Microbiol Infect 15: 977-981.

9. Palefsky J (2006) Human papillomavirus-related tumors in HIV. Curr Opin Oncol 18: 463-468.

10. Qualters JR, Lee NC, Smith RA, Aubert RE (1992) Breast and cervical cancer surveillance, United States, 1973-1987. MMWR CDC Surveill Summ 41: 1-7.

11. Gustafsson L, Pontén J, Zack M, Adami HO (1997) International incidence rates of invasive cervical cancer after introduction of cytological screening. Cancer Causes Control 8: 755-763.

12. Dunleavey $R$ (2005) The role of viruses and sexual transmission in anal cancer Nurs Times 101: 38-41.

13. Centers for Disease Control and Prevention (2009) HIV Surveillance Report 21.

14. Blair JM, Fleming PL, Karon JM (2002) Trends in AIDS incidence and survival among racial/ethnic minority men who have sex with men, United States, 19901999. J Acquir Immune Defic Syndr 31: 339-347

15. Ka'opua LS, Mueller CW (2004)Treatment adherence among Native Hawaiians living with HIV. Soc Work 49: 55-63.

16. Cranston RD, Darragh TM, Holly EA, Jay N, Berry JM, et al. (2004) Selfcollected versus clinician-collected anal cytology specimens to diagnose anal intraepithelial neoplasia in HIV-positive men. J Acquir Immune Defic Syndr 36 915-920.

17. Lampinen TM, Miller ML, Chan K, Anema A, van Niekerk D, et al. (2006) Randomized clinical evaluation of self-screening for anal cancer precursors in men who have sex with men. Cytojournal 3: 4.

18. Minkler M, Wallerstein N (2003) Community based participatory research for health. Jossey-Bass: San Francisco, CA 490.

19. Patton MQ (1990) Qualitative research and evaluation methods. 3 ed London, UK: Sage Publications. 598

20. DeVellis R (1991) Scale Development, Theory and Applications. First ed Applied Social Research Methods Series. Vol. 26. Newbury Park: Sage Publications

21. Maxwell J (2005) Qualitative Research Design, an Interactive Approach Applied Social Research Methods. Vol. 42. London: Sage Publications.
22. Charmaz K(2006) Constructing Grounded Theory, Los Angeles.

23. Chin-Hong PV, Berry JM, Cheng SC, Catania JA, Da Costa M, et al. (2008) Comparison of patient- and clinician-collected anal cytology samples to screen for human papillomavirus-associated anal intraepithelial neoplasia in men who have sex with men. Ann Intern Med 149: 300-306.

24. Solomon D, Davey D, Kurman R, Moriarty A, O'Connor D, et al. (2002) The 2001 Bethesda System: terminology for reporting results of cervical cytology. JAMA 287: 2114-2119.

25. Wright TC Jr, Cox JT, Massad LS, Twiggs LB, Wilkinson EJ, et al. (2002) 2001 Consensus Guidelines for the management of women with cervical cytological abnormalities. JAMA 287: 2120-2129.

26. Gravitt PE, Peyton CL, Alessi TQ, Wheeler CM, Coutlée F, et al. (2000) Improved amplification of genital human papillomaviruses. J Clin Microbiol 38 357-361.

27. Bouvard V, Baan R, Straif K, Grosse Y, Secretan B, et al. (2009) A review of human carcinogens--Part B: biological agents. Lancet Oncol 10: 321-322.

28. Schiffman M, Solomon D (2009) Screening and prevention methods for cervical cancer. JAMA 302: 1809-1810.

29. Chai SJ, Aumakhan B, Barnes M, Jett-Goheen M, Quinn N, et al. (2010) Internet-based screening for sexually transmitted infections to reach nonclinic populations in the community: risk factors for infection in men. Sex Transm Dis 37: 756-763.

30. Cohen J (1960) A coefficient of agreement for nominal scales: a review. Educ Psychol Meas 20: 37-46.

31. Landis JR, Koch GG (1977) The measurement of observer agreement for categorical data. Biometrics 33: 159-174.

32. Branch, H.S.D.o.H.S.A. (1998) An epidemiological profile of HIVIAIDS in Hawai'i. Hawaii State Department of Health: Honolulu.

33. Branch, H.S.D.o.H.S.A. (2001) An epidemiological profile of HIVIAIDS in Hawai'i. Hawaii State Department of Health: Honolulu. 